\title{
Designing Multidimensional Cubes from Warehoused Data and Linked Open Data
}

\author{
Franck Ravat \\ IRIT-Université Toulouse I Capitole \\ UMR5505, CNRS \\ Toulouse, France \\ ravat@irit.fr
}

\author{
Jiefu Song \\ IRIT-Université Toulouse I Capitole \\ UMR5505, CNRS \\ Toulouse, France \\ song@irit.fr
}

\author{
Olivier Teste \\ IRIT-Université Toulouse II Jean Jaurès \\ UMR5505, CNRS \\ Toulouse, France \\ teste@irit.fr
}

\begin{abstract}
A Data Warehouse (DW) is widely used as a consistent and integrated data repository in Business Intelligence systems. Under today's dynamic and competitive business context, warehoused data alone no longer provide enough information for decision-making processes. Business analyses should be enhanced by including Linked Open Data (LOD) to offer multiple perspectives to decision-makers. This paper provides a new multidimensional model, named Unified Cube, which offers a generic representation for both warehoused data and LOD at the conceptual level. A two-stage process is proposed to build a Unified Cube according to decision-makers' needs. As a first step, schemas published with specific modeling languages are transformed into a common conceptual representation. The second step is to associate together related data to form a Unified Cube containing all useful information about an analysis subject. A high-level declarative language is provided to enable nonexpert users to define the relevance between data according to their analysis needs. To demonstrate the feasibility of the proposed concepts, we show how analyses over data from different sources can be carried out through a Unified Cube.
\end{abstract}

Keywords - conceptual multidimensional modeling; linked open data; unified business analyses

\section{INTRODUCTION}

For over a decade, Business Intelligence systems have been widely used to provide complex information during a decision-making process. Operational data are periodically extracted, transformed and loaded in a consistent and integrated repository, called Data Warehouse (DW), which organizes data into multidimensional cubes. Decision-making based only on warehoused data gives a partial view over the activities of the organization. Under today's highly competitive business context, additional information coming from the outside of an organization, mostly found on the Web, should also be included in analyses to provide multiple perspectives to decision-makers [1].

Extending business analyses with external data requires knowing the exact semantics of information. Using semantic web formats, Linked Open Data (LOD) ${ }^{1}$ is designed to publish semantically interconnected and machine-readable data on the Web. By simply accessing data providers, numerous multidimensional LOD can be extracted and used in a decision-making context [2]. However, relevant warehoused

\footnotetext{
${ }^{1}$ http://linkeddata.org
}

data and LOD are scattered in different schemas. During an analysis, decision-makers should navigate among several schemas to gather all useful information. The dispersion of warehoused data and LOD leads to repetitive searches for relevant information among various data sources, which reduces the efficiency of analysis. Furthermore, warehoused data and LOD follow specific models in each domain. The differences between DW and LOD models make it hard for decision-makers to carry out analyses over the two types of data in a unified way, which complicates the analysis tasks.

Facing these issues, our aim is to make full use of all relevant data in a decision-making context. To this end, we represent warehoused data and multidimensional LOD in a unified way and independently of the specific modeling languages of each domain. To facilitate the analysis tasks of decision-makers, the unified representation should only include concepts close to business terms.

In this paper, we describe a generic modeling solution for both warehoused data and LOD. We also define a process of unifying all useful data for analyses. First, we define a conceptual multidimensional model, named Unified Cube, which corresponds to a generic representation of data from several sources relating to one analysis subject. Second, based on the generic modeling language of Unified Cubes, we propose a two-stage process to (a) transform various schemas into a generic conceptual representation and (b) link together relevant data to form a unified schema for all useful data. At the end of the process, decision-makers obtain a Unified Cube containing as much related information as possible to make effective and well-informed decisions. To validate the feasibility of the two propositions, we develop a prototype containing a Unified Cube built upon a relational DW and two online LOD datasets. By illustrating how a Unified Cube can be queried, we demonstrate the feasibility of analyzing both warehoused data and LOD in a unified way.

The rest of the paper is organized as follows. Section 2 introduces a running example of unified analyses over data from multiple data sources. Section 3 presents the multidimensional modeling language composed of conceptual definitions and related graphical notations for Unified Cubes. Section 4 describes the process of building a Unified Cube from different schemas in the DW and LOD domains. Section 5 presents some experimental assessments to demonstrate the feasibility of the proposed concepts. Section 6 
discusses related work on unifying relevant element from different schemas in the fields of DW and LOD.

\section{RUNNING EXAMPLE}

In a company specializing in air conditioning devices, a decision-maker refers to an internal R-OLAP DW to assess the performance of sales staff. The DW relates to an analysis subject (i.e. fact), named Sales, which contains a set of numeric indicators (i.e. measure), namely quantity, unit price and revenue of sales. Each measure can be computed according to three analysis axes (i.e. dimensions): salesman, product and date. In Fig. 1, dimensions are implemented through a set of dimension tables. For instance, the dimension named product is composed of four dimension tables, namely PRODUCT, BRAND, RANGE and SECTOR. Each dimension table represents a granularity level. The dimension tables at lower levels contain one or several foreign keys pointing to the higher levels. For instance, the dimension table $P R O D U C T$ reveals the granularity level $P K e y$ with two foreign keys leading to its parent levels $B R A \overline{N D}$ and $R A N G E$. The fact is implemented with a fact table. The set of foreign keys of a fact table points to the lowest granularity levels of the associated dimensions. For instance, the fact table SALES includes three foreign keys associated with the lowest granularity level (i.e. $P K E Y, S K E Y$ and $D K E Y$ ) of the three related dimensions.

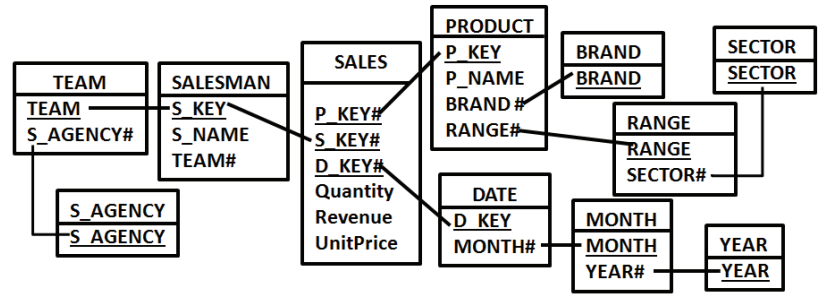

Fig. 1. R-OLAP implementation of the warehoused data.

The R-OLAP DW alone does not provide enough information to support effective and well-informed decisions. The decision-maker must search for additional information to obtain other complementary perspectives over the sales activities. Since she/he notices that the promotion of airconditioning device is more efficient when the customers in a city experience unusual weather changes. To get more insight into the relationships between the promotion activities and the climatic changes, the decision-maker browses in an online LOD dataset revealing the monthly average temperature according to cities and countries during the promotion period of the company. The LOD are published in RDF Data Cube Vocabulary $(\mathrm{QB})^{2}$ format, which is the current W3C standard to publish multidimensional statistical data. In brief, a $\mathrm{QB}$ dataset includes a set of qb:observations ${ }^{3}$ (close to the definition of facts in DW terminology) that describes qb:measureproperty (i.e. measures) with related qb: dimensionProperty (i.e. dimensions). For instance, in Fig. 2 the qb: dataset named Climate Changes includes two instances of qb:observation identified by eg:ob1 and

${ }^{2}$ http://www.w3.org/TR/vocab-data-cube

${ }^{3}$ Details about the prefixes are available on http://prefix.cc/ eg:ob2 (cf. lines 15-24). Each instance of qb : observation relates an instance of eg:M TEMPERATURE with an instance of associated dimensions, namely eg : GEOGRAPHY and eg: TIME.

eg: CLIMATE

a qb:dataset ;

rdfs:label "Climate Changes"@en;

qb:structure :CLIMCHANGE.

eg:CLIMCHANGE a qb:DatastructureDefinition;

rdfs:label "Data Structure"@en;

qb: component

[ qb:dimension eg:TIME],

[ qb:dimension eg:GEOGRAPHY]; $\mathrm{qb}$ : component

[qb:measureproperty eg:M_TEMPERATURE].

eg:M_TEMPERATURE a qb:measureProperty;

rdfs:label "Temperature"@en;

rdfs:range xsd:decimal.

eg:ob1 a qb:Observation; qb: dataset eg:CLIMATE;

eg:TIME eg:ym2015-11;

eg: GEOGRAPHY eg:c1; eg:M_TEMPERATURE 12 .

eg:ob2 a qb:Observation;

qb:dataset eg:CLIMATE;

eg:TIME :ym2015-11;

eg:GEOGRAPHY eg:CO1;

eg:M TEMPERATURE 10 .

Fig. 2. Extract of the LOD published in QB format.

It is worth noticing that unlike the hierarchical definition of dimension in DWs, a dimension in QB is a non-hierarchical concept. The unique granularity level within a QB dimension is represented by skos: hasTopConcept at the schema level. For instance, in Fig. 3 the attributes eg:CITY and eg:COUNTRY share an unspecified granularity level on the dimension eg : GEOGRAPHY (cf. line 29).

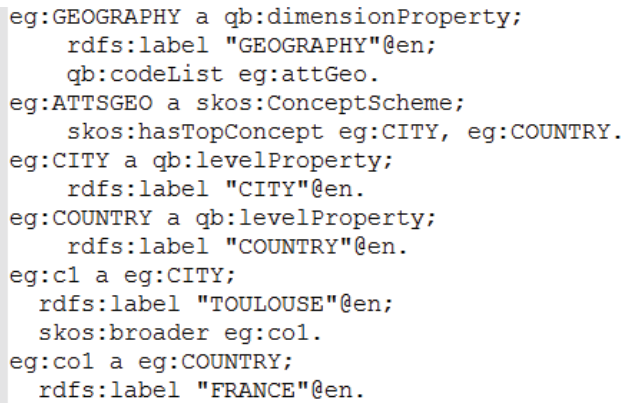

Fig. 3. Dimension GEOGRAPHY in the QB datset.

Since the retail sales may compete with the company's promotions in the same catchment area, the decision-maker consults another online LOD dataset about the outlet prices offered by rival retailers, This dataset is published in QB4OLAP; it involves the retail price for a type of merchandise offered by a retailer. In the field of LOD, QB4OLAP is the only vocabulary that covers the most used characteristics of multidimensional models, like multiple granularity levels (i.e. qb4o:level InHierarchy) within multiple aggregation paths (i.e. qb4o: hierarchyProperty) and the specification of the aggregation functions associated to a measure (i.e. qb4o:aggregateFunction). Even though QB4OLAP covers most features of multidimensional models, the decision-maker has difficulties to explore directly such a schema due to the complex syntax (cf. Fig. 4). 


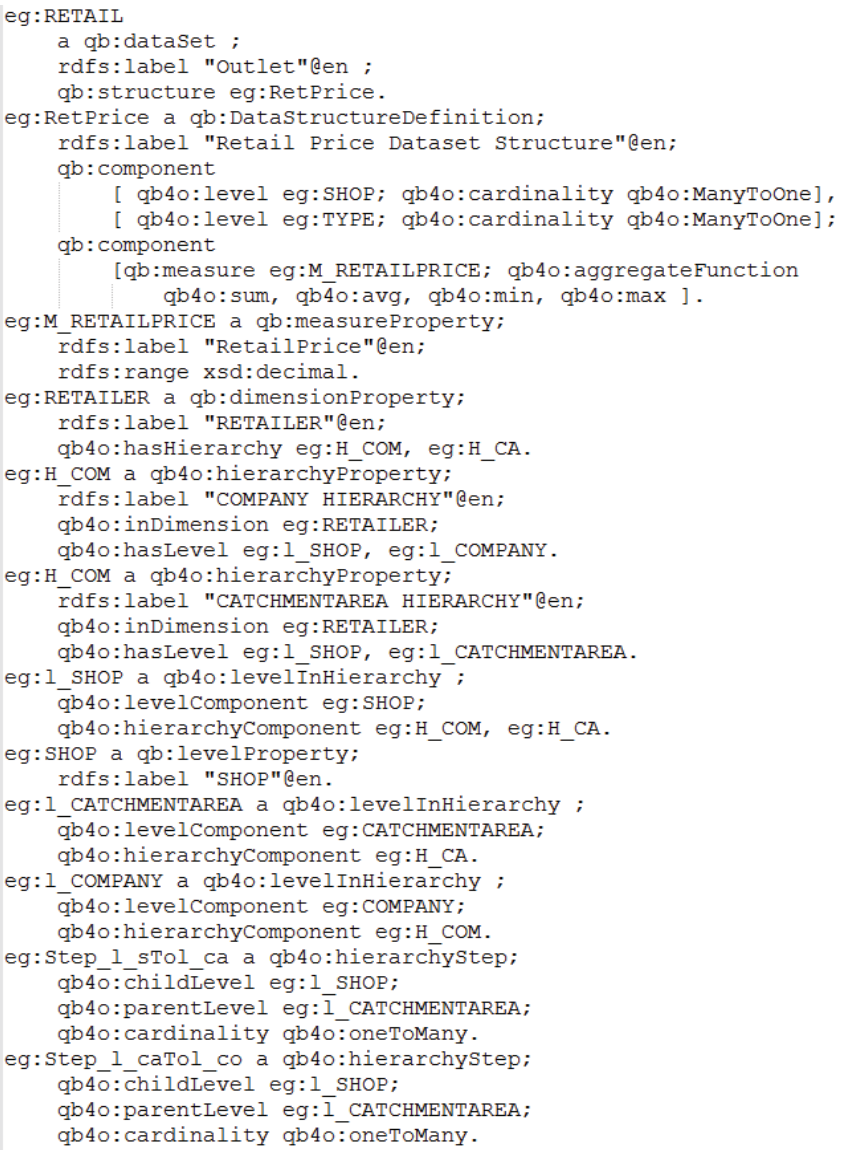

Fig. 4. Extract of the LOD published in QB4OLAP format.

Despite sharing some common features, warehoused data and multidimensional LOD follow different models defined by specific modeling languages in each domain at the logical level [3], [4]. The complex syntax of these modeling languages complicates the tasks of analysis, especially for non-expert users. Moreover, since each of schemas provides a partial view over the analysis subject, the decision-maker should look into several schemas one after another to obtain multiple perspectives. Confronted with these issues, the decision-maker wants to build a conceptual multidimensional schema that includes both the data from the R-OLAP DW and the relevant QB and QB4OLAP datasets.

In the remainder of this paper, we will present how a Unified Cube can help make full use of both warehoused data and LOD in a decision-making context.

\section{CONCEPTUAl Modeling OF UNIFIEd CUBes}

In this section, we describe a multidimensional modeling language composed of a set of conceptual definitions of Unified Cubes. The modeling language is generic enough to include both warehoused data and multidimensional LOD within a single schema.

Besides the differences in the syntax of modeling languages, the communities of DW and LOD do not share the same principles while publishing data: the domain of DW mainly focuses on the structure of data (i.e. schema), while the LOD domain encourages interconnecting independent instances without necessarily being associated to a data schema. In order to represent both warehoused data and LOD, the multidimensional modeling language should include conceptual definitions for components at both schema level and instance level. Graphical notations are also proposed to facilitate the understanding of non-expert users.

\section{A. Dimension Schema}

A dimension schema represents the structure of an analysis axis composed of attributes organized in one or multiple aggregation levels. The definition of dimension schemas should be generic enough to cooperate with different DW and LOD models containing (a) multiple attributes within a level, (b) mono-hierarchical dimensions, (c) multiplehierarchical dimensions and (d) non hierarchical dimensions.

Definition 1. A dimension schema is a triple $D_{i}=\left\langle\mathrm{dn}^{\mathrm{i}}\right.$, $\left.\mathcal{L}^{\mathrm{i}}, \prec^{\mathrm{i}}\right\rangle$, such as:

- $\mathrm{dn}^{\mathrm{i}}$ is the dimension's name;

- $\mathcal{L}^{\mathrm{i}}$ is a finite set of pairs of $\left\langle l_{\mathrm{x}}, \mathcal{A}_{\mathrm{x}}\right\rangle$, such that $l_{\mathrm{x}}$ is a level and $\mathcal{A}_{\mathrm{x}}$ is a set of attributes associating to a level;

o among the levels, there exist at least a unique root level and a unique extreme level;

$\circ$ among the attributes of a level, the unique attribute identifying the level is called parameter, denoted $p_{\mathrm{x}}$, while the other attributes describing the semantics of the parameter are called weak attribute, denoted Weak $k_{\mathrm{x}}, W e a k_{\mathrm{x}}=\mathcal{A}_{\mathrm{x}} \backslash\left\{p_{\mathrm{x}}\right\}$. The domain of an attribute $\mathrm{a}_{\mathrm{k}}$ $\left(\mathrm{a}_{\mathrm{k}} \in \mathcal{A}_{\mathrm{x}}\right)$ is denoted as $\operatorname{dom}\left(\mathrm{a}_{\mathrm{k}}\right)$.

- $<\mathrm{i}$ is a set of asymmetric and transitive binary relations which reveals the aggregation path between a pair of levels. Remember that the asymmetry means that $\exists l_{\mathrm{m}}$, $l_{\mathrm{n}}, l_{\mathrm{k}} \in \mathcal{L}^{\mathrm{i}}:\left(l_{\mathrm{m}} \prec^{\mathrm{i}} l_{\mathrm{n}}\right) \wedge\left(l_{\mathrm{n}} \prec^{\mathrm{i}} l_{\mathrm{m}}\right) \Rightarrow l_{\mathrm{m}}=l_{\mathrm{n}}$, while the transitivity means that $\left(l_{\mathrm{m}}<l_{\mathrm{n}}\right) \wedge\left(l_{\mathrm{n}}<{ }_{\mathrm{k}}^{\mathrm{i}}\right) \Rightarrow l_{\mathrm{m}} \prec^{\mathrm{i}} l_{\mathrm{k}}$.

Remark. In the case of a non hierarchical dimension (e.g. dimensions in a QB schema), the root level contains all nonextreme attributes, while the set of binary relations only includes the one between the root level and the extreme level.

We propose the following graphical notation of dimension schema for non-expert users (cf. Fig. 5). For simplicity, the extreme level is omitted in the graphical notation.

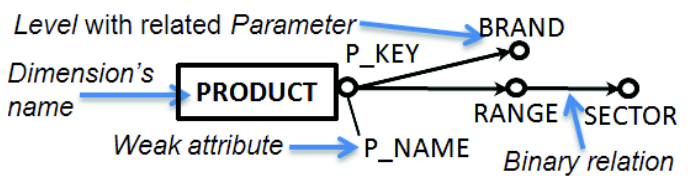

Fig. 5. Graphical notation of a dimension schema.

In the remainder of this paper, we abusively note $\mathcal{L}$ and $\prec$ instead of $\mathcal{L}^{\mathrm{i}}$ and $\prec^{\mathrm{i}}$ when it is clear that $\mathcal{L}$ and $\prec$ represents the element of the dimension $\mathrm{D}$ in question.

\section{B. Dimension Instance}

The conceptual definition of dimension instances is required to ensure the interoperability between schemacentered DW models and instance-centered LOD models. A 
dimension instance (a) maps the parameter at each level with the corresponding parameter instances, (b) describes the hierarchical aggregation relationships between parameter instances and (c) associates the values of a weak attribute to the related parameter instance.

Definition 2. A dimension instance consists of:

- a function between an attribute $a_{\mathrm{k}}\left(a_{\mathrm{k}} \in \mathcal{A}_{\mathrm{x}}\right)$ at the level $l_{\mathrm{x}}$ $\left(l_{\mathrm{x}} \in \mathcal{L}\right)$ and its instances (i.e. any constant in $\left.\bigcup_{l_{\mathrm{x}} \in \mathcal{L}} \pi_{l_{\mathrm{x}}}(\mathrm{D})^{4}\right)$;

- a rollup function revealing the part-whole relationship between a child attribute and a parent attributes, denoted $\Uparrow_{p_{\mathrm{x}}}^{p_{\mathrm{y}}}$, from $p_{\mathrm{x}}$ to $p_{\mathrm{y}}, \operatorname{such}$ that $l_{\mathrm{x}}<l_{\mathrm{y}}, \Uparrow_{p_{\mathrm{x}}}^{p_{\mathrm{y}}}: \operatorname{dom}\left(p_{\mathrm{x}}\right) \rightarrow \operatorname{dom}\left(p_{\mathrm{y}}\right)$;

- an association function $\operatorname{Map}_{p_{\mathrm{x}}}^{W e a k_{\mathrm{x}}}$ mapping an instance of the parameter $p_{\mathrm{x}}$ to the values of its weak attributes $W e a k_{\mathrm{x}}$, such that $\operatorname{Map}_{p_{\mathrm{x}}}^{\text {Weak }}{ }_{\mathrm{x}}: \operatorname{dom}\left(p_{\mathrm{x}}\right) \rightarrow 2^{\mathrm{U}_{\mathrm{a}_{\mathrm{k}} \in \text { Weak }_{\mathrm{x}}} \operatorname{dom}\left(\mathrm{a}_{\mathrm{k}}\right)}$.

\section{Unified Cube Schema}

A Unified Cube schema represents the multidimensional structure of data coming from one or multiple sources. Related dimensions from different sources are linked together, so that decision-makers can obtain new perspectives and more complete information during analyses.

Definition 3. An Unified Cube schema is denoted as $\langle\mathrm{ucn}$, $\mathcal{D}, \mathcal{M}$, ExtLink, such as:

- ucn is the name of the Unified Cube;

- $\mathcal{D}=\left\{\mathrm{D}_{1} ; \ldots ; \mathrm{D}_{\mathrm{n}}\right\}$ is a finite set of dimensions;

- $\mathcal{M}=\left\{\mathrm{m}_{1} ; \ldots ; \mathrm{m}_{\mathrm{k}}\right\}$ is a finite set of numeric indicators called measures;

- ExtLink is a set of extrinsic links depicting the relevance between data. An extrinsic link is an interdimension mapping which associates together two disparate levels on different dimensions. The starting point of an extrinsic link is called mapping level, while the end point is called mapped level.

Remark. The definition of Unified Cube schemas is generic enough to represent the structure of data from one or multiple sources. In the case where only one data source is involved, we obtain a Unified Cube without extrinsic links (i.e. ExtLink $=\varnothing)$. If multiple sources are included in a Unified Cube, a set of extrinsic links should be built to associate relevant data together. Details about different types of extrinsic link will be discussed in the section IV.B.

Fig. 6 shows the graphical notation of Unified Cube built upon the warehoused data. Measures sharing the same related dimensions are grouped together within the graphical notation.

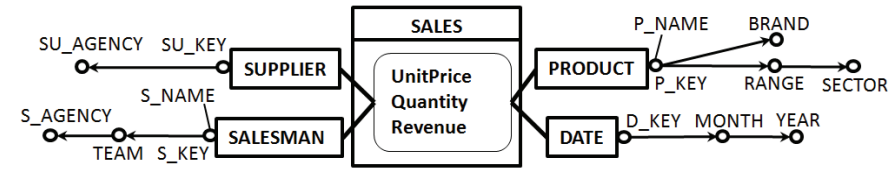

Fig. 6. Graphical notation of a Unified Cube with only warehoused data.

\footnotetext{
${ }^{4} \pi$ represents the projection operator of the relational algebra
}

\section{Unified Cube Instance}

A Unified Cube instance (a) associates each measure value to the instances of parameters on the related dimensions and (b) connects the parameter instances at the mapping level with relevant ones at the mapped level.

Definition 4. Let $\left\{p_{1}, \ldots, p_{\mathrm{n}}\right\}$ be a set of parameters, in which $p_{\mathrm{x}}$ is a parameter of the dimension $\mathrm{D}_{\mathrm{x}}, \operatorname{dom}\left(\mathrm{m}_{\mathrm{j}}\right)$ is the values of the measure $\mathrm{m}_{\mathrm{j}}$, a Unified Cube instance consists of:

- a function between a set of parameter instances and the related measure values, such as $\forall \mathrm{m}_{\mathrm{j}} \in \mathcal{M}: 2^{\mathrm{U}_{\mathrm{x} \in[1, \mathrm{n}]} \operatorname{dom}\left(p_{\mathrm{x}}\right)}$ $\rightarrow \operatorname{dom}\left(\mathrm{m}_{\mathrm{j}}\right)$;

- an instance of extrinsic link revealing the relevance between data. Each extrinsic link instance associates the parameter instances at a mapping level $l_{\mathrm{i}}$ with the relevant parameter instances at a mapped level $l_{\mathrm{j}}$, such as $\operatorname{dom}\left(p_{\mathrm{i}}\right) \rightarrow \operatorname{dom}\left(p_{\mathrm{j}}\right)^{5}$.

\section{Process of BuIlding A Unified CubE}

Based on the generic multidimensional modeling language of Unified Cubes, warehoused data and LOD can be unified together in a single schema. In this section, we present a twostage process allowing building a Unified Cube from multiple sources in the domains of DW and LOD.

As is shown in Fig. 7, the first step is to transform data schemas published with specific modeling vocabularies into a generic conceptual representation with a common modeling language. The multidimensional modeling language of Unified Cube can be used in the step 1, since it is generic enough to cover all the features embedded in multidimensional models. The conceptual representation obtained after the first step is called exportation cube whose aim is to facilitate the combination of different schemas in a Unified Cube.

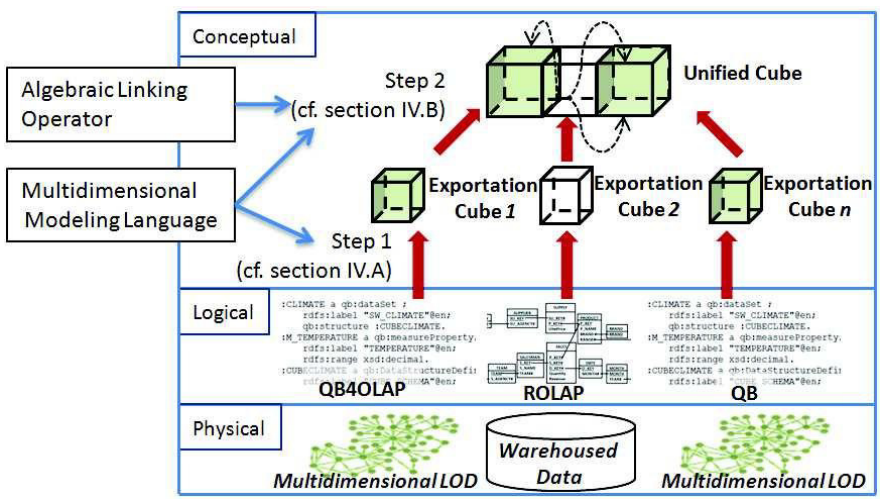

Fig. 7. Building a Unified Cube from multiple data sources.

The second step aims at linking together relevant data to obtain a unified schema. To do so, the multidimensional modeling language of Unified Cube provides a component, named extrinsic link, which allows representing the relevance between two dimensions. To facilitate the linking operations for non-expert users, we propose a high-level declarative language in the form of algebraic operator which translates a

\footnotetext{
${ }^{5} \sigma$ represents the selection operator in the relational algebra
} 
decision-maker's need into a mapping between two relevant dimensions. An algorithm is also proposed for the linking operator to automate its execution and guarantee the overall validity of a Unified Cube .

The outcome of the process is a Unified Cube which represents all useful data for analyses at the conceptual level along with the relevance between different dimensions.

\section{A. Step I: Generic Conceptual Representation}

Warehoused data and LOD are published according to different models. Each data model follows a specific modeling language expressed with the terminology of the corresponding domain. The differences between various modeling languages in DW and LOD domains make it impossible to analyze all useful data in a unified way. To overcome this problem, different data sources should be firstly transformed into a generic representation with a common modeling language.

The modeling language of Unified Cube is rich enough in expressivity to cover the complete multidimensional features embedded in OLAP, QB and QB4OLAP schemas (cf. section III). As the first step of building a Unified Cube, different data sources are transformed into a generic conceptual representation, named exportation cube, which aims at facilitating the combination of all useful data in a Unified Cube. No user intervention is needed for the first step. An exportation cube is automatically obtained by referring to the translation rules presented in this section.

\section{1) From an OLAP Schema to an Exportation Cube}

The transformation of an OLAP schema into a conceptual representation has been thoroughly studied during the last decade [5]. Since the modeling language of Unified Cube covers all multidimensional features of OLAP models, the transformation of an OLAP schema into exportation cube is quite straightforward and thus does not need to be repeated in this paper. The graphical notations of the obtained exportation cube of the R-OLAP DW (cf. Fig. 1) can be found in Fig. 6.

\section{2) From a QB4OLAP Schema to an Exportation Cube}

As an extension of QB vocabulary, the main idea of QB4OLAP is to use RDF triples to represent the most common features of a multidimensional model. More specifically, QB4OLAP adds classes and properties (prefixed by $q b 4 o$ ) to represent multiple attributes (i.e. a parameter and its weak attributes) within a level, hierarchical dimensions with multiple levels and the set of aggregate functions associated to a measure. TABLE I. gives an overview of the conceptual definitions of exportation cubes and their corresponding QB4OLAP triples. Note that in the domain of LOD, a variable starts with a question mark.

TABLE I. UNIFIED CUBE CONCEPTS AND QB4OLAP TRIPLES

\begin{tabular}{|l|l|}
\hline $\begin{array}{c}\text { Unified Cube } \\
\text { Concept }\end{array}$ & \multicolumn{1}{c|}{ QB4OLAP triples } \\
\hline $\begin{array}{l}\text { Dimension } \\
\text { schema (?dim) }\end{array}$ & ?dim a qb:DimensionProperty; \\
\hline Level (?lvl) & $\begin{array}{l}\text { ?dim qb4o: hasHierarchy ?hierarchy; } \\
\text { ?hierarchy a qb40: hierarchyProperty; } \\
\text { ?hierarchy qb40: hasLevel ?lvl. }\end{array}$ \\
\hline
\end{tabular}

\begin{tabular}{|c|c|}
\hline $\begin{array}{l}\text { Unified Cube } \\
\text { Concept }\end{array}$ & QB4OLAP triples \\
\hline $\begin{array}{l}\text { Parameter } \\
\text { (?para) } \\
\text { weak attribute } \\
\text { (?att) }\end{array}$ & $\begin{array}{l}\text { ?lvl a qb4o: level InHierarchy; } \\
\text { ?lvl qb4o: levelComponent ?para; } \\
\text { ? para a qb: level Property; } \\
\text { ?para qb4o: hasAttribute ?att. }\end{array}$ \\
\hline $\begin{array}{l}\text { Binary Relation } \\
\text { (?biRel) }\end{array}$ & $\begin{array}{l}\text { ?biRel a qb4o: hierarchystep; } \\
\text { ?biRel qb4o: childLevel ?lvlChild; } \\
\text { ?biRel qb4o: parentLevel ?lvlParent; } \\
\text { ?biRel qb4o: cardinality ?cardinalityParentChild }\end{array}$ \\
\hline $\begin{array}{l}\text { Dimension } \\
\text { Instance } \\
\text { (?dimIns, } \\
\text { ?dimInsParent }\end{array}$ & $\begin{array}{l}\text { ?dimIns qb4o: inLevel ?lvlChild; } \\
\text { ?dimIns rdfs : label ?InsChild; } \\
\text { ?dimIns skos : broader ?dimInsParent. } \\
\text { ?dimInsParent qb4o: inLevel ?lvlParent; } \\
\text { ?dimInsParent rdfs : label ?InsParent. }\end{array}$ \\
\hline $\begin{array}{l}\text { Cube Schema } \\
\text { (?cube) }\end{array}$ & $\begin{array}{l}\text { ?cube a qb: dataSet; } \\
\text { ?cube rdfs: label ?cubeName; } \\
\text { ?cube qb: structure ?cubeStructure; } \\
\text { ?cubeStructure a qb: DataStructureDefinition; } \\
\text { ?cubeStructure qb: component [qb4o:level ?lvlRoot; } \\
\quad \text { qb } 40: \text { cardinality ?cardinalityCubeDim]; } \\
\text { ?cubeStructure qb: component [qb:measure?measure]. }\end{array}$ \\
\hline $\begin{array}{l}\text { Measure } \\
\text { (?measure) }\end{array}$ & $\begin{array}{l}\text { ?measure a qb: measureProperty; } \\
\text { ?measure rdfs: label ?mName. }\end{array}$ \\
\hline $\begin{array}{l}\text { Cube Instance } \\
(? \mathrm{ob})\end{array}$ & $\begin{array}{l}\text { ?ob a qb: Observation; } \\
\text { ?ob qb: dataset ?cube; } \\
\text { ?ob ?dim ?dimIns; } \\
\text { ?ob ?measure ?value. }\end{array}$ \\
\hline
\end{tabular}

Based on the TABLE I. we translate the LOD dataset about the outlet sales into an exportation cube. Its graphical notations are shown in Fig. 8.

\section{3) From a $Q B$ Schema to an Exportation Cube}

$\mathrm{QB}$ is the current W3C standard to publish statistical LOD. In terms of multidimensional features, QB allows defining the structure of a fact via qb:DatastructureDefinition. However, it does not support the same hierarchical structure of dimension as a multidimensional model does. In a multidimensional model, a dimension schema corresponds to a hierarchical structure whose attributes are organized according to multiple granularity levels. A dimension schema defined in QB, on the contrary, may only contain one granularity level with a non-hierarchical list of attributes.

One way to find the hierarchical relationship between two attributes within a $\mathrm{QB}$ dimension is to look into dimension instances. However, obtaining a hierarchical dimension schema from a QB dataset is not straight-forwards as it seems to be. For instance, the property skos: hasTopConcept (or qb: hierarchyRoots in the case of non-skos hierarchies) is, by convention, used to link a dimension schema to the topmost attribute in a hierarchy. However, on the contrary to a hierarchy in a multidimensional schema, there is no integrity constraint enforcing the top-most convention in a QB schema. By consequence, publishing the following dimension schema, which is strictly forbidden according to a multidimensional model, is nevertheless consistent with the QB specifications.

eg:dimSchema skos:hasTopConcept eg:oneAtt eg:oneAttribute skos:broader eg:anotherAtt eg:anotherAtt skos:inscheme eg:dimschema

Facing these issues, we propose the following algorithm to obtain an exportation cube from a QB dataset. 


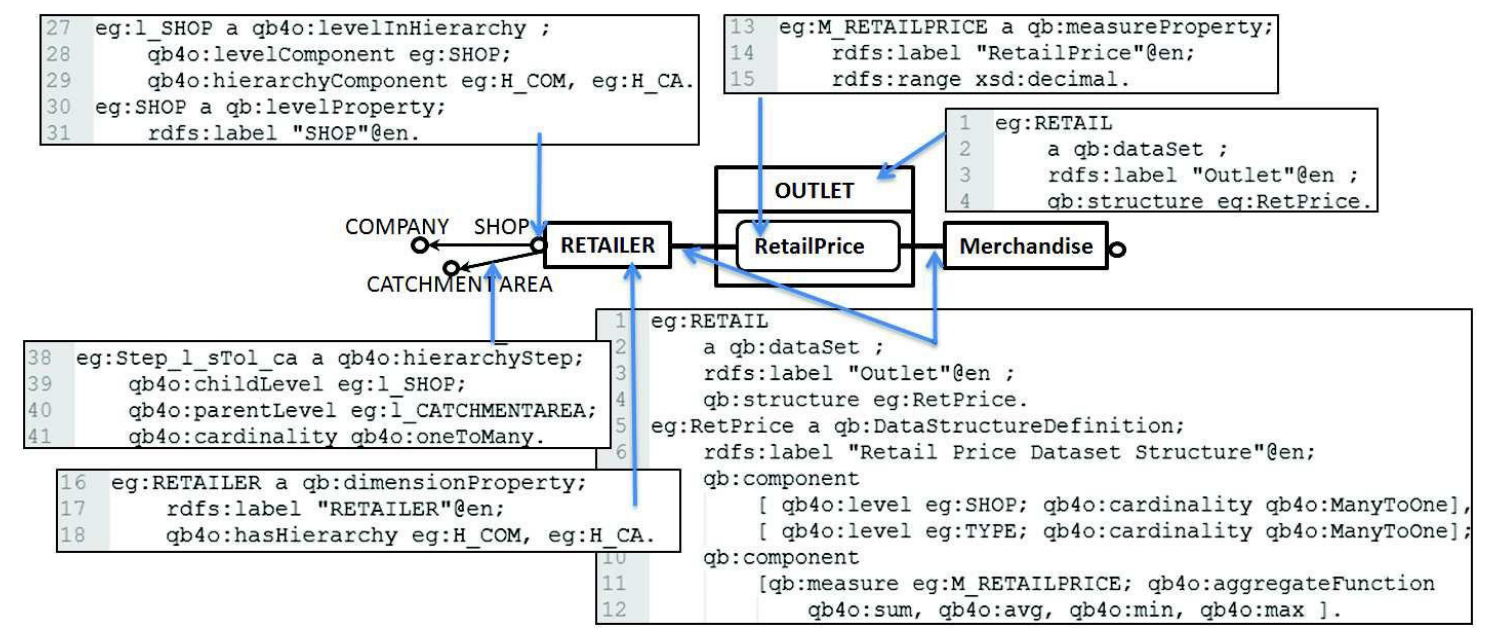

Fig. 8. Exportation Cube for LOD in QB4OLAP format.

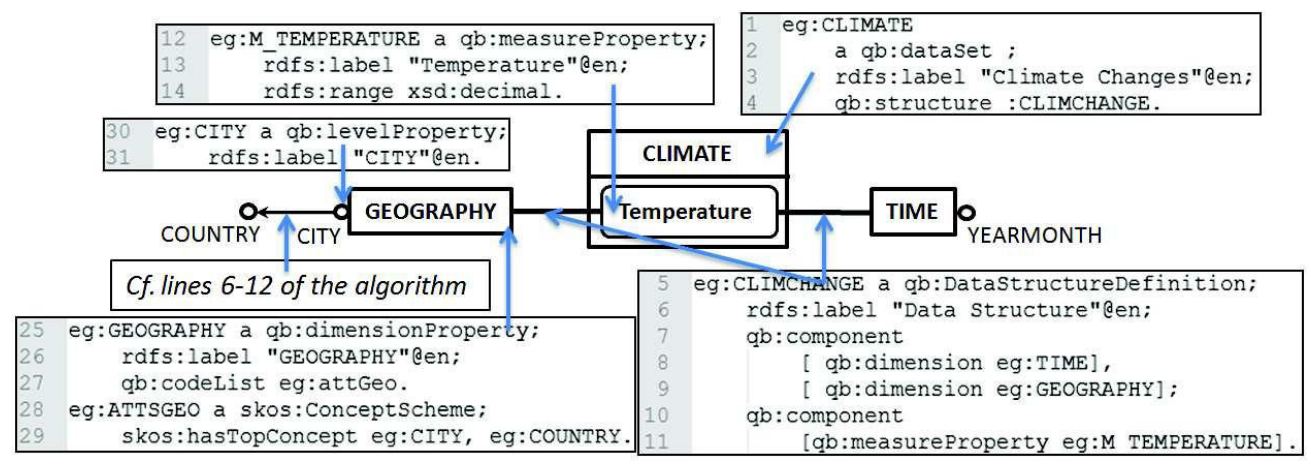

Fig. 9. Exportation Cube for LOD in QB4OLAP format.

Algorithm Creating an exportation cube from a QB schema Input: A QB schema qbCube where cubeStructure is the stucture of the schema; $D_{\mathrm{QB}}$ is the set of dimensions; $M_{\mathrm{QB}}$ is the set of measures.

Output: A conceptual exportation cube.

\section{Begin}

1. For each $d_{\mathrm{i}} \in D_{\mathrm{QB}}\left(d_{i}\right.$ a qb: dimensionProperty)

2. Create a dimension with the name $\operatorname{dim} N$ such as $d_{\mathrm{i}}$ rdfs : label $\operatorname{dim} N$;

3. For each attribute $a_{\mathrm{k}}$ of $d_{\mathrm{i}}\left(d_{\mathrm{i}}\right.$ qb: codeList $c l_{\mathrm{i}}$; $c l_{\mathrm{i}}$ a

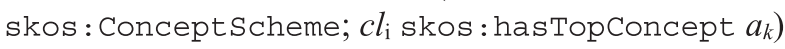

4. $\quad$ Create a level $l_{\mathrm{k}}$ for $a_{\mathrm{k}}$;

5. Associate $\left\langle l_{\mathrm{k}}, a_{\mathrm{k}}\right\rangle$ to $d_{\mathrm{i}}$;

6. For all attribute instances $I_{\mathrm{k}}$ of $a_{\mathrm{k}}\left(I_{\mathrm{k}}\right.$ a $\left.a_{\mathrm{k}}\right)$

7. If $I_{\mathrm{k}}$ skos : broader $I_{\mathrm{k}+1}\left(I_{\mathrm{k}+1}\right.$ a $\left.a_{\mathrm{k}+1}\right)$

8. Create a binary relation $l_{\mathrm{k}}<l_{\mathrm{k}+1}$;

9. $\quad$ ElseIf $I_{\mathrm{k}}$ skos : narrower $I_{\mathrm{k}-1}\left(I_{\mathrm{k}-1}\right.$ a $\left.a_{\mathrm{k}-1}\right)$

10. Create a binary relation $l_{\mathrm{k}-1}<l_{\mathrm{k}}$;

11. End If

12. End for

13. End For

14. End For

15. Create an exportation cube named cubeN such as $q b C u b e$ a qb: dataset; $q b C u b e$ rdfs: label cubeN;

16. For each $m_{\mathrm{j}} \in M_{\mathrm{QB}}$ ( $m_{\mathrm{j}}$ a $\mathrm{qb}:$ measureProperty)

17. Create a measure in the exportation cube with the name
meN such as $m_{\mathrm{j}}$ rdfs : label $m e N$;

18. Associate this measure with its associated dimensions $D_{m j} \subseteq D_{\mathrm{QB}}, \quad$ such as $\forall d_{\mathrm{x}} \in D_{m j}$ : cubeStructure a qb: DatastructureDefinition; cubeStructure qb: component [qb:dimension $d_{\mathrm{x}}$ ], qb: component [q] : measureProperty $m_{\mathrm{j}}$ ] ;

19. End for

End.

Remark. As the authors of [6] mention, the QB format is not always correctly used in some real-world application cases. To avoid bringing inaccurate information to decision-makers, error-detection methods such as those presented in [7] should be applied to a QB schema before applying the proposed algorithm.

We apply the algorithm to the QB dataset about climate changes. The obtained exportation cube is shown in Fig. 9. After each data source is transformed into a corresponding exportation cube, we regroup together all exportation cubes in a preliminary Unified Cube. The preliminary Unified Cube aims at providing a unified view of all useful data for analyses, so that decision-makers can associate relevant data together during the second step of the process.

\section{B. Step II: Cube Linking}

The multidimensional modeling language of Unified Cubes includes relationships between data within one source and inter-schema relationships representing the relevance 
between data from disparate sources (i.e. extrinsic links). In the previous sections, we discuss how different relationships embedded in one data source can be automatically transformed into exportation cubes. The aim of this section is to present how inter-schema relationships are established and managed in a Unified Cube, which corresponds to the second step of the process. First, we describe two types of extrinsic links that a decision-maker can define between two related dimensions. Second, we propose a high-level declarative language in the form of algebraic operator, named DLink, which builds extrinsic links between relevant data according to decision-makers' needs. An algorithm is proposed to automate the execution of the DLink operator while ensuring the validity of the built Unified Cube.

\section{1) Types of Extrinsic Links}

The relevance between data from disparate sources may take many forms. Among different types of relevance, the correlation and the aggregation relations are particularly useful in the context of business analysis.

\section{a) Correlation Links}

Classically an analysis granularity includes only one level of dimension. In the context of Unified Cubes, the notion of analysis granularity needs to be generalized, since several levels of dimension from different data sources may refer to the same analysis granularity. To this end, we propose correlation links which associate together two relevant levels that belong to the same analysis granularity.

A correlation link allows associates a pair of levels on two dimensions that are considered equivalent in term of analytical needs. The parameters involved in the levels associated by a correlation link may be semantically equivalent (i.e. referring to the same concept), such as the parameter named YearMonth in the QB dataset and the one named Month in the R-OLAP DW (cf. Fig. 10(a)). In the graphical notation of a Unified Cube, correlation links are represented by dotted lines.
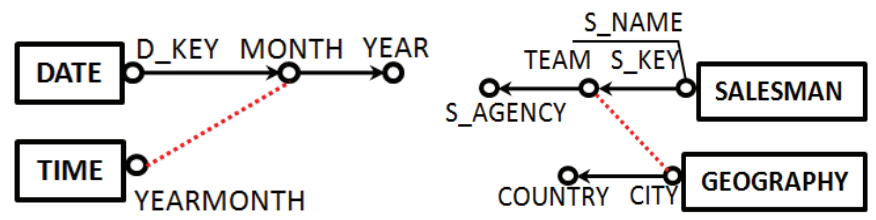

(a) Semantically equivalent elements (b) Analytically equivalent elements

Fig. 10. Correlation links at the schema level.

Two levels that are semantically heterogeneous but share the same role in business analyses (i.e. analytically equivalent) may also be connected together through a correlation link. For instance, the salesmen's team and the city are two semantically heterogeneous concepts. However, in a specific analysis context where each team is in charge of sales in one city, analyses involving a team may as well be carried out with the corresponding city. In this case, the salesmen's team and the city can be considered as two analytically equivalent concepts, which can also be connected together through a correlation link (cf. Fig. 10(b)).

\section{b) Aggregation Links}

Classical multidimensional schemas allow decisionmakers to carry out analyses only according to aggregation paths situated within one dimension. Within a Unified Cube, a level within a dimension may be aggregated to another level on a different dimension. To provide additional aggregation paths for analyses, we propose aggregation links which reveal the parent-child relation between two relevant levels on different dimensions.

An aggregation link gathers parts into a whole by associating one or several instances of the parameter at the mapping level to at most one parameter instance at the mapped level. An aggregation link may be inferred through the semantics embedded in the corresponding parameters. For instance, in Fig. 11(a) since each Type of merchandise may correspond to several product (i.e. $P_{-} K e y$ ), we can build an aggregation link between the levels $l_{\text {Type }}$ and $l_{P_{\mathrm{P} K e y}}$. An aggregation link is represented by a dotted arrow in the graphical notation of a Unified Cube.

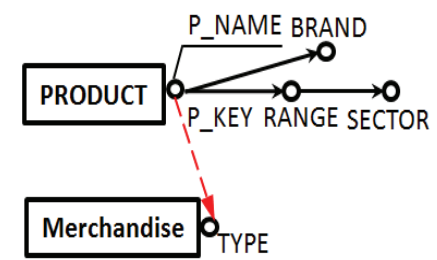

(a) Semantic parent-child relation

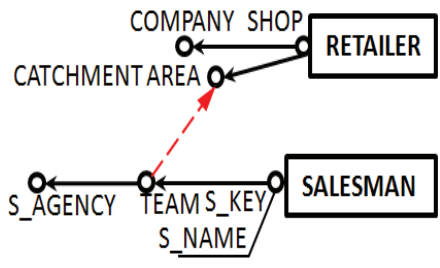

(b) Analytical parent-child relation
Fig. 11. Aggregation links at the schema level.

In the previous case, the aggregation link represents the parent-child relation between a product and a type which can be deduced independently of the analysis context. An aggregation link may also be placed between two levels whose parent-child relations holds only under a given analysis context. For instance, the aggregation link in Fig. 11(b) between salesman's team and retailer's catchment area cannot be detected through the semantics embedded in the parameters. It is valid only under a specific analysis context where a catchment area of a retailer attracts the same clientele of the nearby salesman's teams.

\section{2) Establishing Extrinsic Link}

As one of the key characteristics of the next generation of BI, the internal complexity of a system should be hidden from end-users. In the context of a Unified Cube, it comes down to empower non-expert users with the ability to associate by themselves relevant data together according to their needs. Ideally, decision-makers could be able to express their needs through a high-level declarative language to a system. To this end, we define an user-oriented linking operator presented in an algebraic form, named DLink. The DLink operator allows associating relevant dimensions and producing a Unified Cube of all useful information as output. An algorithm is proposed to automate the execution of the DLink operator and ensure the validity of the obtained Unified Cube.

\section{a) Algebraic Linking Operator}

The DLink operator builds an extrinsic link according to a specified type between a pair of levels on two dimensions. TABLE II. shows the algebraic representation of DLink. 
TABLE II. ALGEBRAIC DLINK OPERATOR

\begin{tabular}{|c|c|}
\hline \multicolumn{2}{|c|}{ DLink $\left(\mathrm{D}_{\text {mapping }} ; l_{\text {mapping }} ; \mathrm{D}_{\text {mapped }} ; l_{\text {mapped }} ;\right.$ Type $)=$ Unified Cube } \\
\hline Input & $\begin{array}{l}-\mathrm{D}_{\text {mapping: }}: \text { the starting dimension of the extrinsic link; } \\
-l_{\text {mapping: }} \text { the starting level of the extrinsic link; } \\
-\mathrm{D}_{\text {mapped }} \text { the ending dimension of the extrinsic link; } \\
-l_{\text {mapped }} \text { the ending level of the extrinsic link; } \\
- \text { Type }=\{\text { correlation link; aggregation link }\}: \text { the type } \\
\text { of extrinsic link to establish between levels. }\end{array}$ \\
\hline Output & $\begin{array}{l}\text { Unified Cube: a Unified Cube with an updated set of } \\
\text { extrinsic links. }\end{array}$ \\
\hline
\end{tabular}

At the schema level, the DLink operator associates a pair of levels with a correlation link or an aggregation link. At the instance level, different mapping functions are created between a parameter instance at the mapped level and the corresponding one(s) at the mapping level.

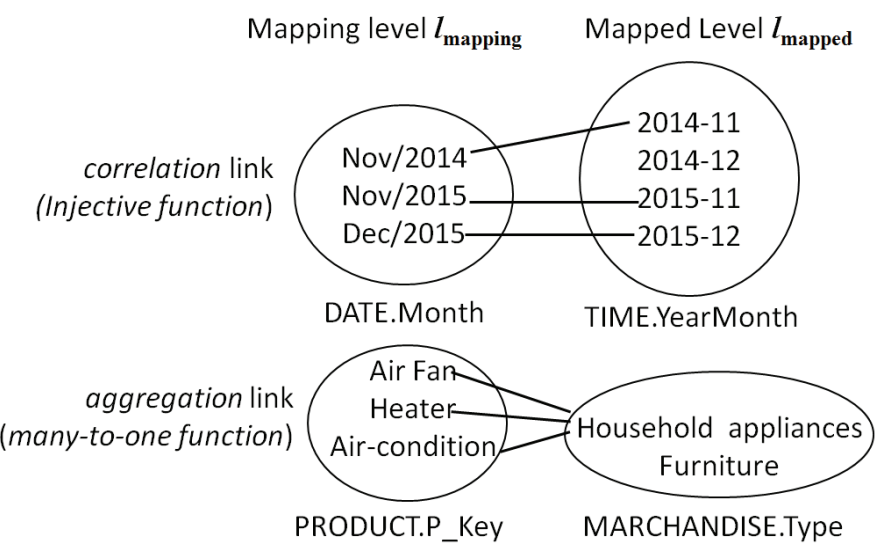

Fig. 12. Extrinsic links at the schema level and mappings at the instance level.

As is shown in Fig. 12, the correlation link corresponds to an injective function between the mapping level and the mapped level. The injection implies an instance of the parameter at the level $l_{\text {mapping }}$ is mapped to at most one instance of the parameter at the level $l_{\text {mapped}}$. The correlation link in Fig. 12 is built via the following operator: $\boldsymbol{D} \boldsymbol{L} \boldsymbol{i n k}\left(\mathrm{D}_{\mathrm{DATE}}\right.$; $l_{\text {Month }}$; $\mathrm{D}_{\mathrm{TIME}} ; l_{\text {YearMonth; }}$; correlation link $\}$ ).

The aggregation link is a many-to-one function between the mapping level and the mapped level. A many-to-one function associates one or several child instances at the level $l_{\text {mapping }}$ to at most one parent element at the level $l_{\text {mapped }}$ (cf. Fig. 12). For instance, the aggregation link associating one or several products to their corresponding type is built through the following operator: $\boldsymbol{D} \boldsymbol{L} \boldsymbol{i n k}\left(\mathrm{D}_{\mathrm{PRODUCT}}\right.$; $l_{\mathrm{P}_{-} \mathrm{Key}}$; $\mathrm{D}_{\text {MARCHANDISE}}$; $l_{\text {Type; }}$ \{aggregation link\}).

\section{b) Execution Algorithm of the DLink Operator}

To facilitate the tasks of non-expert users, we propose an algorithm to automate the execution of the DLink operator. Two types of operations are included in the algorithm, namely dimension mapping and validity verification. The dimension mapping operations aim at creating automatically or semiautomatically an extrinsic link between a pair of relevant levels on two dimensions, while the validity verification operation aim at removing inconsistent or redundant extrinsic links in the new Unified Cube. The execution algorithm is as follows.

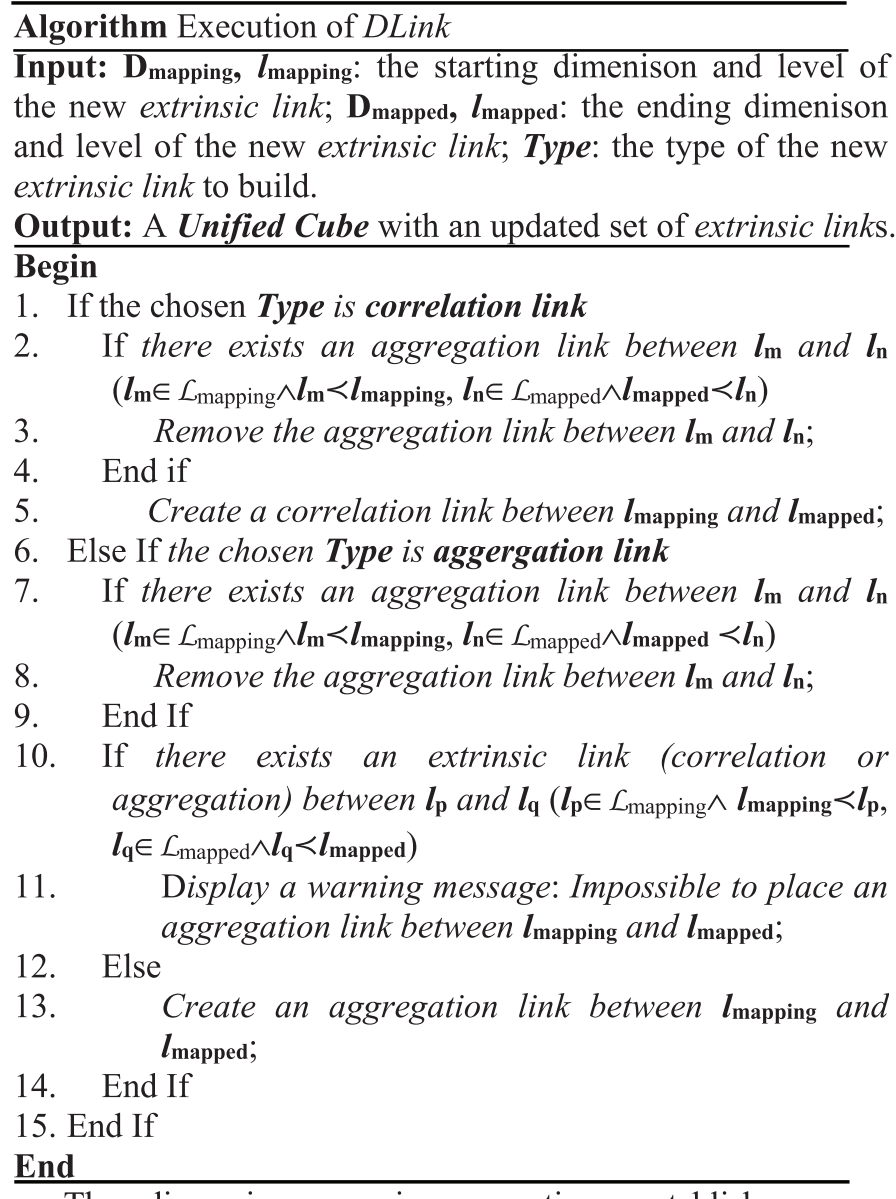

The dimension mapping operations establish a new extrinsic link between relevant dimensions (cf. lines 5 and 13). During the dimension mapping operations, relevant dimensions are associated together at both schema level and instance level. If the two dimensions correspond semantically (e.g. Date and Time, Product and Merchandise), the execution of dimension mapping operations may be automated. In this case, we may refer to the approaches presented in [8] to automatically establish mappings at the schema level as well as instance level. When two dimensions are relevant only under certain analysis contexts (e.g. Salesman and Retailer, Salesman and Geography), a semi-automatic approach should be adopted. In this case, the system proposes to decisionmakers several possibilities of mappings between two dimensions based on domain ontology, semantic annotations or integrity constraints [9], [10]. Then decision-makers can choose or modify a proposed mapping according to a given analysis context.

Through the dimension mapping operations, decisionmakers can associate, according to their analysis needs, relevant dimensions within a Unified Cube. At the same time, the algorithm of the DLink operator must make sure the global validity of all extrinsic links in the new Unified Cube, especially when each new extrinsic link seems to be valid if being built individually. To do so, the algorithm carries out 
validity verification operations to check the validity of the new extrinsic link and the existing ones. Decision-makers do not need to worry about the order in which the set of extrinsic links should be placed, because inconsistent or redundant extrinsic links are automatically removed from the Unified Cube after the validity verification operations.

More specifically, the algorithm checks if the new extrinsic link can replace a redundant extrinsic link already existing in a Unified Cube. Firstly, a correlation link can substitute an aggregation link built with a lower mapping level and/or a higher mapped level (cf. lines 2-4 of the following algorithm). For instance, suppose there was an aggregation link between the level $l_{\text {YearMonth }}$ of the dimension TIME and the level $l_{\text {Year }}$ on the dimension DATE. Once the correlation link between $l_{\text {YearMonth }}$ and $l_{\text {Month }}$ (cf. Fig. 10(a)) is built, the aggregation link between $l_{\text {YearMonth }}$ and $l_{\text {Year }}$ should be removed from the Unified Cube, since it can be deduced from the new correlation link along with the binary relation $l_{\text {Month }}<l_{\text {Year. }}$

Similarly, an aggregation link can replace another aggregation link built with a lower mapping level and/or a higher mapped level (cf. lines 7-9 of the following algorithm). For instance, building the aggregation link between $l_{\text {Team }}$ and $l_{\text {CatchmentArea }}$ would replace an aggregation link between $l_{\mathrm{S} \text { Key }}$ and $l_{\text {CatchmentArea, }}$, because the latter can be deduced from the new aggregation link and the binary relation $l_{\mathrm{S} \_ \text {Key }}<l_{\text {Team }}$ (cf. Fig. 11(a)).

On the other hand, a new aggregation link should not be built if a correlation link or an aggregation link already exists at a higher mapping level and/or a lower mapped level in the same Unified Cube (cf. lines 10 and 11 of the algorithm). For instance, since there is already a valid correlation link between $l_{\text {City }}$ and $l_{\text {Team }}$ (cf. Fig. 10(b)), no more aggregation link is allowed between $l_{\text {City }}$ and $l_{S_{-} \text {Agency }}$ since it can be deduced from the correlation link and the binary relation $l_{\text {Team }}<l_{\text {S_Agency. }}$.

After the execution of four DLink operators, two correlation links and two aggregation links are built within the Unified Cube as shown in the Fig. 13.

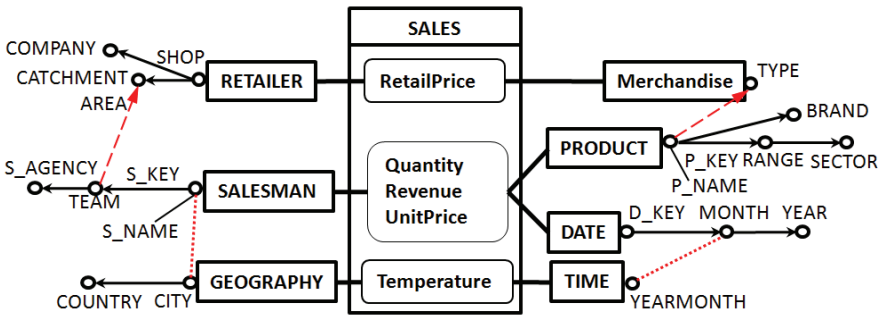

Fig. 13. Unified Cube for warehoused data and LOD

\section{EXPERIMENTAL ASSESSMENTS}

In this section, we carry out some experimental assessments to demonstrate the feasibility of analyzing both warehoused data and LOD in a unified way. The advantage of unified analyses is that decision-makers can obtain complete and coherent information about an analysis subject and new analysis possibilities by following the relationships established between relevant data from different sources.

\section{A. Protocol}

\section{1) Configuration for Data Implementation}

To simulate the distributed nature of warehoused data and LOD, we use three identical Microsoft Windows 7 work stations located on the same LAN for the experimental assessments (Interl(R) i7-4510U 2GHz CPU, 8GB RAM, SSD 500GB disk). Each work station hosts one source of the running example: the DBMS Oracle $11 \mathrm{~g}$ manages the warehoused data implemented with a snowflake schema, while a native triple store with an integrated SPARQL endpoint provided by Apache Jena API [11] is used for LOD published in QB and QB4OLAP formats. Several warm-up runs are carried out in each work station after the installation. We implement the Unified Cube in a virtual machine installed on the same work station hosting the R-OLAP DW. Only one dedicated core of CPU, 500M RAM and 1GB disk are allocated to the virtual machine, since managing a Unified Cube is not resource-consuming; it only requires hosting a non-materialized view for the Unified Cube and an ontology implementing the set of extrinsic links by associating together relevant data at the instance level.

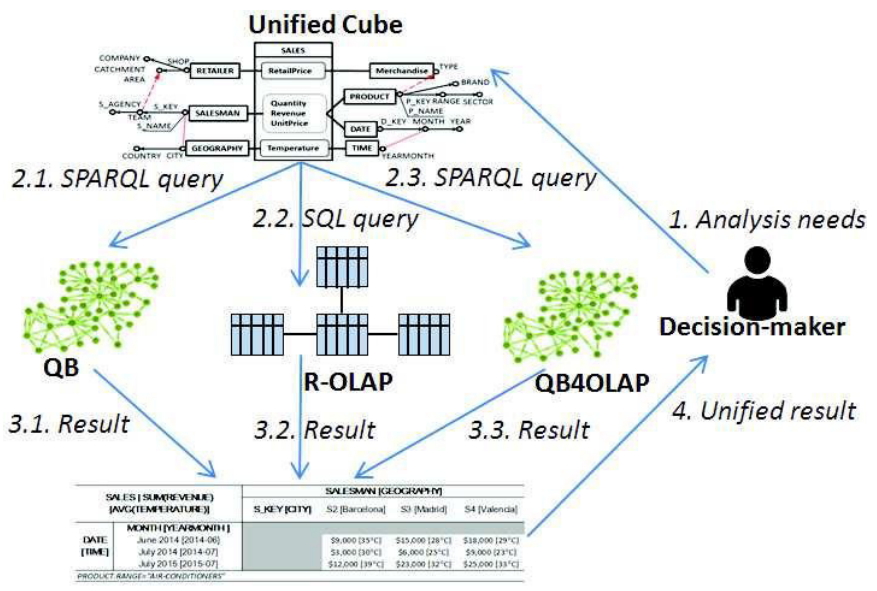

Fig. 14. Procedures for processing analyses in a Unified Cube.

An analysis need over a Unified Cube is translated into one or several queries which are applied to the corresponding component data sources. The analysis processing procedures are as follows (cf. Fig. 14). First, by identifying all useful measures and attributes in a Unified Cube, a decision-maker expresses an analysis need. This analysis need is manually divided into a set of user-oriented algebraic operators. Each algebraic operator involves data from one source. Two existing tools with graphical interface developed within our research team [12], [13] are used to automate the translation of an algebraic operator into an executable query over a R-OLAP DW or a LOD dataset. Then, each of the three work stations receives a SQL or SPARQL query and produces a partial result based on the hosted source. Each partial result is cleaned up, especially for SPARQL queries in which the URI of each returned triple is simplified to keep only useful information for analyses. At last, we query the ontology implementing the extrinsic links to associate relevant data scattered among the partial results. In this way, a unified result is created before being presented to decision-makers. 


\section{2) Queries and Data Collection}

To support analyses over both warehoused data and LOD, different types of queries should be correctly generated and executed in a Unified Cube. We carry out analyses covering the most widely used types of queries in the context of multidimensional analyses, i.e. queries containing joins, projections, selection criteria and aggregation functions with grouping clauses (i.e. Group By). More details are available in TABLE III.

TABLE III. ANALYSIS NEEDS, QUERY TyPES AND DATA SOURCES

\begin{tabular}{|c|c|c|}
\hline Analysis Need & Query & Source \\
\hline $\begin{array}{l}\text { 1. Find the sale quantities for heaters (product } \\
\text { range) according to the country in which the } \\
\text { salesmen promote. }\end{array}$ & $\begin{array}{l}\text { Join, } \\
\text { Select }\end{array}$ & $\begin{array}{c}\text { R-OLAP } \\
\text { QB }\end{array}$ \\
\hline $\begin{array}{l}\text { 2. Find the average price and quantity of products } \\
\text { sold by each salesman's team, associating the price } \\
\text { with the average outlet retail price for the products } \\
\text { of the same type offer by the retailers in the } \\
\text { corresponding catchment area. }\end{array}$ & $\begin{array}{l}\text { Join, } \\
\text { Selection, } \\
\text { Projection } \\
\text { Group By }\end{array}$ & $\begin{array}{l}\text { R-OLAP, } \\
\text { QB4OLAP }\end{array}$ \\
\hline
\end{tabular}

In consideration of the computing capacity of the three work stations, we populate the three datasets with a reasonable amount of synthetic data to avoid query execution timeout. The R-OLAP DW contains over 16 million tuples while the QB and QB4OLAP schemas respectively include about 1.44 million and 1.21 million triples ${ }^{6}$.

\section{B. Result and Discussion}

In this section, we present and discuss the results of our experimental assessments. To separate the fundamental operations from the complex syntax of querying languages, the generated SQL and SPARQL queries are presented in the form of relational algebra and SPARQL algebra ${ }^{7}$ respectively. Note that although the SPARQL algebra has not yet become a W3C standard, it is already supported by several frameworks for querying LOD: all algebraic expressions of SPARQL queries in this section are generated by Apache Jena API.

The first analysis corresponds to a cross-source analysis which calculates measures from one data source according to parameters in another data source. During this analysis, the Unified Cube offers additional perspectives of analysis to decision-makers by aggregating sale quantities from R-OLAP DW according to the parameter named Country from the QB dataset. This cross-source analysis is feasible within the Unified Cube owning to the correlation link between $l_{\mathrm{S} \text { Key }}$ on $\mathrm{D}_{\text {Salesman }}$ and $l_{\text {City }}$ on $\mathrm{D}_{\text {Geography }}$ as well as the binary relation $l_{\text {City }}<l_{\text {Country }}$ within the dimension Salesman .

Two queries are generated for the first analysis (cf. Fig. 15). The SQL query searches for sale quantities of heaters by salesmen, while the SPARQL query returns the list of countries in the QB dataset.

\footnotetext{
${ }^{6}$ The source code and generated queries are available at http://olap-sw.weebly.com/blog/rcis-2016-source-code-andqueries

${ }^{7}$ https://www.w3.org/2001/sw/DataAccess/rq23/rq24algebra.html
}

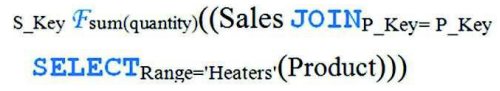

(a) SQL Query

(PROJECT (?country)

(BGP

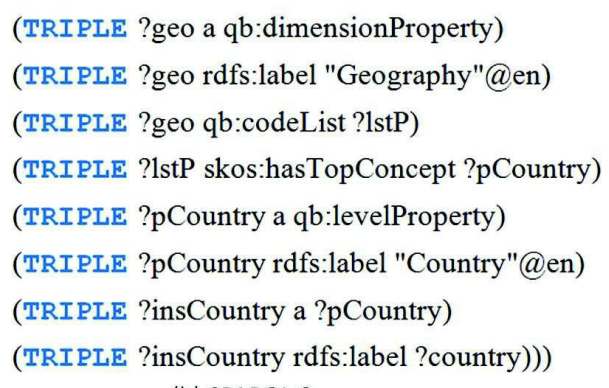

(b) SPARQL Query

Fig. 15. Generated SQL and SPARQL queries for the first analysis.

After querying the ontology implementing the extrinsic links, relevant instances of parameters $S_{-}$Key and City are associated together. Then, by referring to the rollup function between the instances of City and Country, a country is associated with a corresponding group of salesmen within the unified analysis result which regroups the results of SQL and SPARQL queries. Through the result shown in Fig. 16, the decision-maker realizes that the salesmen who have difficulty in selling the heaters are in fact located in hot countries where the demand of heaters remains relatively low. Without the Unified Cube, it would be difficult to obtain multiple perspectives in such an intuitive way if the analysis was carried out with separate data sources.

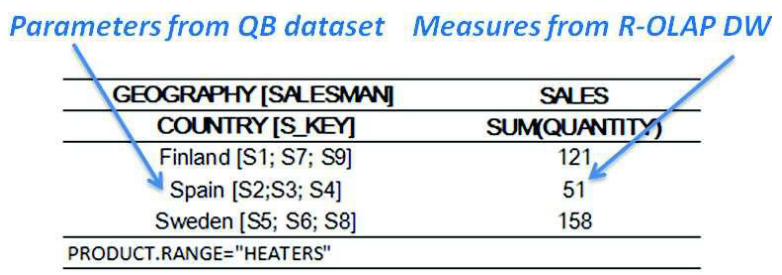

Fig. 16. Unified result of the first analysis.

The second analysis corresponds to a comparative analysis which requires including relevant information from different data sources in one analysis result. A Unified Cube allows displaying measures from different sources according to related dimensions which are interconnected through extrinsic links. During the second analysis, the measures quantity and unit price from the R-OLAP DW can be displayed along with the retail price from the QB4OLAP dataset starting from the levels associated by the aggregation link between $\mathrm{D}_{\text {Product }}$ and $\mathrm{D}_{\text {Merchandise }}$ as well as the one between $\mathrm{D}_{\text {Salesman }}$ and $\mathrm{D}_{\text {Retailer. }}$

The second analysis need is translated into a SQL query and a SPAQL query (cf. Fig. 17). The SQL query searches for the unit prices and the sale quantities by products and salesman' teams, while the SPARQL query reveals the retail price of the same product's type offered by retailers in the catchment areas sharing the same clientele of salesman's teams. 
Team, P_Key $F_{\text {avg(unitPrice), avg(quantity) }}\left(\left(\right.\right.$ Sales JOIN S_KEY $_{-}$_KEY Product)

(a) SQL Query

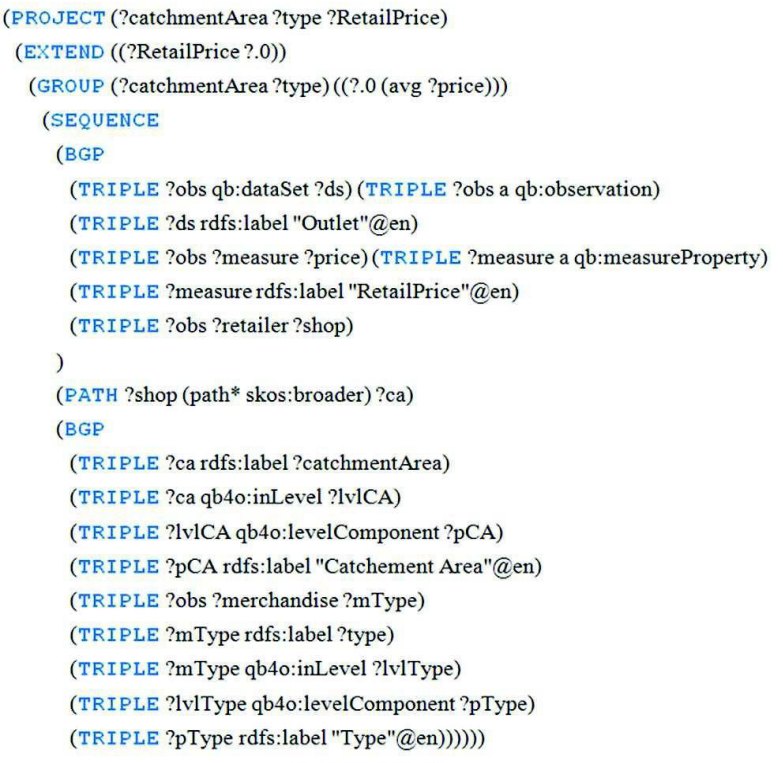

(b) SPARQL Query

Fig. 17. Generated SQL and SPARQL quereis for the second analysis.

After the execution of these two partial queries, each product is associated with its corresponding type through the aggregation link between $l_{P_{-} \text {Key }}$ on $D_{\text {Product }}$ and $l_{\text {Type }}$ on

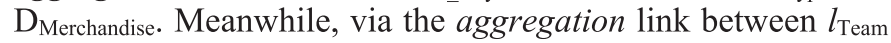
on $\mathrm{D}_{\text {Salesman }}$ and $l_{\text {CatchmentArea }}$ on $\mathrm{D}_{\text {Retailer }}$ each salesman's team is aggregated to the related catchment area which shares the same clientele of the company. The unification of relevant dimensions makes it possible to combine measures from $\mathrm{R}$ OLAP DW with the one from QB4OLAP dataset. By comparing with the price offered by other retailers (cf. Fig. 18), the decision-maker notices that the higher the sale prices fixed by the company, the lower the sale quantities promoted by the salesmen. It would be less easy to obtain such a comprehensive analysis result without gathering relevant data in dispersed sources into a Unified Cube.

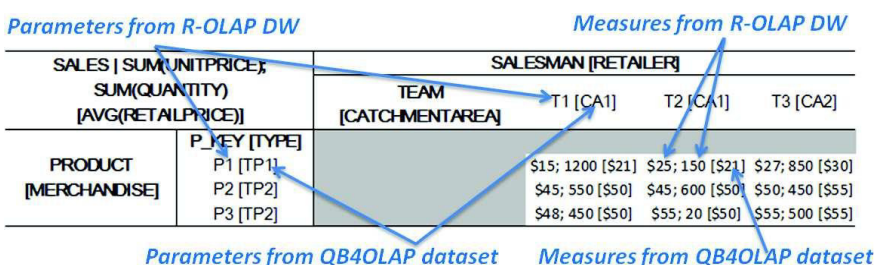

Fig. 18. Unified result of the second analysis.

We record the execution time of each analysis from the moment where an analysis need is submitted from the decision-maker till the moment where a unified result is produced by gathering all partial results. Without any optimization technique for query execution, all analyses return the result within one minute (cf. TABLE IV. column Execution Time). The execution time remains reasonable in consideration of the laptop-level configuration of the working stations.
TABLE IV. EXECUTION TIME OF EACH ANALYSIS

\begin{tabular}{|c|c|c|c|c|}
\hline \multirow{2}{*}{$\begin{array}{l}\text { Analysis } \\
\text { Need }\end{array}$} & \multirow{2}{*}{$\begin{array}{l}\text { Execution Time } \\
\text { (Seconds) }\end{array}$} & \multicolumn{3}{|c|}{ Working Station's Response Time (Seconds) } \\
\hline & & $R-O L A P$ & $Q B$ & QB4OLAP \\
\hline 1 & 29.8 & 7.9 & 27 & $\mathrm{n} / \mathrm{a}$ \\
\hline 2 & 56.5 & 8.5 & $\mathrm{n} / \mathrm{a}$ & 51.0 \\
\hline
\end{tabular}

A Unified Cube increases the efficiency of analysis by allowing decision-makers to analyze all useful data in a unified way. The execution time of each analysis is much lower than the sum of response time of all working stations, which is impossible if decision-makers query different data sources separately and wait for all partial results one after another. Meanwhile, each working station receives and executes a partial query individually without any influence on others. Thus, another advantage of Unified Cubes is the possibility of parallelizing the execution of partial queries, which helps further reduce the execution time of analysis.

\section{RELATED WORK}

In today's highly dynamic business context, well-informed and effective decisions require enriching warehoused data with other data that fall outside of the scope of internal DWs [1], [14]. Establishing relationships between elements from different sources belongs to the field of schema matching. Being studied for the first time as an independent research topic in [15], schema matching refers to a set of techniques allowing generating correspondences between several formal structures that represent an engineered artifact. Within the scope of this paper (i.e. the fields of DW and LOD), the classical application cases of schema matching include ETL and ontology alignment [8].

In the DW domain, an ETL (extract-transform-load) process integrates one or several disparate data sources (e.g. operational databases) into the warehouse format. With the arrival of web published data in business analyses, the DW community intuitively treated LOD as external data sources that should be centralized in a DW through an ETL process [16], [17]. The shortcoming of this approach was found soon afterwards due to the poor freshness of warehoused LOD and the high cost of non-automatic ETL process [18]. To overcome these drawbacks, [6] and [19] propose solutions to querying directly LOD published in QB or other multidimensional formats derived from QB. Among the derived LOD formats, the QB4OLAP vocabulary allows representing the complete multidimensional structure of LOD at the logical level [4]. However, the previous work is limited to representing LOD from one data source with a multidimensional structure expressed through semantic web languages. No solution is proposed to deal with the problems about how disparate data sources can be represented and analyzed in a unified way. In this paper, we define a generic conceptual model allowing including both warehoused data and LOD. Differing from the semantic web approaches whose main goal is fixing a common vocabulary and a set of interpretation constraints (i.e., inferring rules) to describe the data semantics, our model aims at including all useful data in a generic and unified multidimensional schema.

In the field of semantic web, ontology alignment is the de facto application case of schema matching [20], [21]. Ontology alignment allows matching semantically relevant 
elements in different schemas. In term of practical utility for business analyses, ontology alignment is not generic enough to represent common analytical relationships that are semantically irrelevant. For instance, in our running example the statement "a salesman's team is a equivalent concept to city in an analytical context about the sales of air-conditioners" cannot be represented through any standardized ontology property without violating the strict logical semantics of identity: by no means team and city may refer to the same concept in terms of semantic. Our work is more generic since both semantically and analytically relevant data can be associated together according to decision-makers' needs.

\section{CONCLUSION AND DISCUSSIONS}

Our aim is to make full use of all relevant data to support effective and well-informed decisions. To this end, we define a generic multidimensional model, named Unified Cube, which allows including as much useful information as possible for analyses. The multidimensional modeling language of Unified Cubes includes both conceptual definitions and graphical notations.

To build a Unified Cube, we describe a two-stage process which unifies relevant data from disparate sources. As a first step, schemas published with specific modeling languages in the DW and LOD domains are transformed into a common conceptual representation named exportation cube. Defined through a generic multidimensional modeling language, exportation cubes allow facilitating the combination of useful data in a Unified Cube. The second step of building a Unified Cube is to associate relevant data according to decisionmakers' needs. The multidimensional modeling language includes a set of mappings, named extrinsic links, which allows modeling inter-dimension analysis granularities (i.e. correlation links) and parent-child relations between levels on different dimensions (i.e. aggregation links). An algebraic linking operator named DLink is proposed to enable nonexpert users to establish extrinsic links according to their needs. We propose an algorithm to automate the execution of the DLink operator while guaranteeing the overall validity of all extrinsic links within a Unified Cube.

To demonstrate the feasibility of the proposed concepts, we develop a prototype including a Unified Cube built from a R-OLAP DW and two LOD datasets published in QB and QB4OLAP formats. By translating analysis needs into queries applicable to Unified Cubes, we show how warehoused data and LOD can be analyzed in a unified way.

One of our ongoing research efforts is focused on an analysis framework compatible with Unified Cubes. Following an automatic approach of federated queries processing, this analysis framework aims at enabling non-expert users to carry out on-the-fly analyses including multiples data sources within a Unified Cube. We also intend to generalize the definition of extrinsic links to represent more sophisticated analysis needs. For instance, an n-ary link will be defined to model the relevance between three or more dimensions. A more longterm objective is to study the influences of the materialization of source data over the efficiency of analyses carried out in Unified Cubes.

\section{References}

[1] A. Abelló, O. Romero, T. B. Pedersen, R. Berlanga, V. Nebot, M. J. Aramburu, and A. Simitsis, "Using Semantic Web Technologies for Exploratory OLAP: A Survey," IEEE Trans. Knowl. Data Eng., vol. 27, no. 2, pp. 571-588, Feb. 2015.

[2] M. E. Zorrilla, J.-N. Mazón, Ó. Ferrández, I. Garrigós, F. Daniel, and J. Trujillo, Eds., Business Intelligence Applications and the Web: Models, Systems and Technologies. IGI Global, 2012.

[3] C. Ciferri, R. Ciferri, L. Gómez, M. Schneider, A. Vaisman, and E. Zimányi, "Cube Algebra: A Generic User-Centric Model and Query Language for OLAP Cubes," Int. J. Data Warehous. Min., vol. 9, no. 2, pp. 39-65, 322013.

[4] L. Etcheverry, A. Vaisman, and E. Zimányi, "Modeling and Querying Data Warehouses on the Semantic Web Using QB4OLAP," in Data Warehousing and Knowledge Discovery, vol. 8646, Cham: Springer International Publishing, 2014, pp. 45-56.

[5] S. Chaudhuri and U. Dayal, "An overview of data warehousing and OLAP technology," ACM SIGMOD Rec., vol. 26, no. 1, pp. 65-74, Mar. 1997.

[6] B. Kämpgen and A. Harth, "Transforming statistical linked data for use in OLAP systems," in Proceedings of the 7th international conference on Semantic systems, Graz, Austria, 2011, pp. 33-40.

[7] D. Fleischhacker, H. Paulheim, V. Bryl, J. Völker, and C. Bizer, "Detecting Errors in Numerical Linked Data Using Cross-Checked Outlier Detection," in The Semantic Web - ISWC 2014, vol. 8796, Cham: Springer International Publishing, 2014, pp. 357-372.

[8] E. Rahm, "Towards Large-Scale Schema and Ontology Matching," in Schema Matching and Mapping, Springer Berlin Heidelberg, 2011, pp. $3-27$.

[9] A. DOAN and A. Y. HALEVY, "Semantic integration research in the database community: A brief survey.," AI Mag., vol. 26, no. 1, pp. 8394, 2005.

[10] G. Cabanac, C. Max, F. Ravat, and O. Teste, "Decisional Annotations: Integrating and Preserving Decision-Makers' Expertise in Multidimensional Systems," in Complex Data Warehousing and Knowledge Discovery for Advanced Retrieval Development: Innovative Methods and Applications, IGI Global, 2009, pp. 65-81.

[11] J. J. Carroll, I. Dickinson, C. Dollin, D. Reynolds, A. Seaborne, and K. Wilkinson, "Jena: implementing the semantic web recommendations," in Proceedings of the 13th international World Wide Web conference, New York, NY, USA, 2004, pp. 74-83.

[12] R. Saad, O. Teste, and C. Trojahn, "OLAP Manipulations on RDF Data following a Constellation Model," in Proceedings of International Workshop on Semantic Statistics (SemStats 2013) collocated with International Semantic Web Conference (ISWC-2013), Sydney, 2013.

[13] F. Ravat, O. Teste, R. Tournier, and G. Zurfluh, "Algebraic and Graphic Languages for OLAP Manipulations," Int. J. Data Warehous. Min., vol. 4, no. 1, pp. 17-46, 2008.

[14] A. Abelló, J. Darmont, L. Etcheverry, M. Golfarelli, J.-N. Mazón, F. Naumann, T. Pedersen, S. B. Rizzi, J. Trujillo, P. Vassiliadis, and G. Vossen, "Fusion Cubes: Towards Self-Service Business Intelligence," Int. J. Data Warehous. Min., vol. 9, no. 2, pp. 66-88, 322013.

[15] E. Rahm and P. A. Bernstein, "A survey of approaches to automatic schema matching," VLDB J., vol. 10, no. 4, pp. 334-350, Dec. 2001.

[16] V. Nebot, R. Berlanga, J. M. Pérez, M. J. Aramburu, and T. B. Pedersen, "Multidimensional Integrated Ontologies: A Framework for Designing Semantic Data Warehouses," in Journal on Data Semantics $X I I I$, vol. 5530, Springer Berlin Heidelberg, 2009, pp. 1-36.

[17] O. Romero and A. Abelló, "Automating multidimensional design from ontologies," in international workshop on Data warehousing and $O L A P, 2007$, pp. 1-8.

[18] L. Etcheverry and A. A. Vaisman, "Enhancing OLAP Analysis with Web Cubes," in The Semantic Web: Research and Applications, vol. 7295, Springer Berlin Heidelberg, 2012, pp. 469-483.

[19] L. Etcheverry, S. Gomez, and A. Vaisman, "Modeling and Querying Data Cubes on the Semantic Web," ArXiv Prepr. ArXiv151206080, 2015.

[20] P. A. Bernstein, J. Madhavan, and E. Rahm, "Generic schema matching, ten years later," Proc. VLDB Endow., vol. 4, no. 11, pp. 695-701, 2011.

[21] J. Euzenat, Ontology matching, 2nd edition. New York: Springer, 2013. 